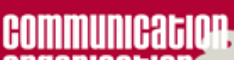

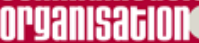

Communication et organisation

Revue scientifique francophone en Communication

organisationnelle

$50 \mid 2016$

Engagement entrepreneurial et territoires

\title{
Développement de marque personnelle en ligne et influence sociale
}

Development of personal brand on-line and social influence

\section{Stéphane Amato}

\section{CpenEdition}

\section{Journals}

Édition électronique

URL : http://journals.openedition.org/communicationorganisation/5429

DOI : 10.4000/communicationorganisation.5429

ISBN : 979-10-300-0127-3

ISSN : $1775-3546$

Éditeur

Presses universitaires de Bordeaux

Édition imprimée

Date de publication : 1 décembre 2016

Pagination : 203-214

ISBN : 979-10-300-0069-6

ISSN : 1168-5549

Référence électronique

Stéphane Amato, « Développement de marque personnelle en ligne et influence sociale »,

Communication et organisation [En ligne], 50 | 2016, mis en ligne le 01 décembre 2019, consulté le 16 février 2021. URL : http://journals.openedition.org/communicationorganisation/5429 ; DOI : https:// doi.org/10.4000/communicationorganisation.5429 


\title{
Développement de marque personnelle en ligne et influence sociale
}

\author{
Stéphone Amato'
}

\section{Introduction: De la «marque personne» à la "personne marque»}

Les grandes agences de publicité se différencient souvent par leurs stratégies de création, éléments formalisés censés permettre, entre autres, la délimitation d'un cadre de travail visant à orienter la création des messages publicitaires. Dans les années quatre-vingt, Jacques Séguéla conçoit le modèle de la "star-stratégie ». Une marque doit pouvoir être décomposée en un physique (une compétence objective, un avantage concurrentiel), un caractère (une qualité durable d'ordre imaginaire), et un style (la façon variable d'exprimer le caractère). Il est possible de voir se dessiner les traits d'un individu et le publicitaire parle d'ailleurs de " marque personne ». Les marques deviennent alors des personnes et il ne manque plus qu'une âme aux barils de lessive et autres objets inanimés.

En période de crise économique, le terme de «marché de l'emploi » prend un sens renouvelé. Les individus sont mis en compétition pour accéder à une situation professionnelle où beaucoup sont appelés mais très peu sont élus. C'est dans ce contexte que le web 2.0 a vu se développer des Réseaux Sociaux Numériques Professionnels (RSNP) qui favorisent notamment la mise en relation des étudiants, des professionnels, des entreprises, et d'accéder à des opportunités d'emploi. Le phénomène est récent mais une proportion non négligeable des recrutements se fait déjà via les réseaux sociaux, selon Cécile Desjardins ${ }^{2}$.

\footnotetext{
1 Stéphane Amato est docteur en Sciences de l'Information et de la Communication, chercheur associé au laboratoire IRSIC, EA 4262, Aix-Marseille Université. Ses travaux de recherche s'articulent principalement autour de trois axes : médias et espaces numériques, communication d'action et d'utilité sociétales, médiation symbolique et rituelle; stephane@amato.fr

2 Cécile DESJARDINS, «Métiers du chiffre : $7 \%$ des recrutements via les réseaux sociaux». Les Échos Business, 2015. Disponible sur http://business.lesechos.fr/directions-financieres/comptabiliteet-gestion/audit/021407038152-7-des-recrutements-se-font-grace-aux-reseaux-sociaux-203975. php?EMpSrfQYKfWEyVj3.99, consulté le 22 octobre 2015.
} 
L'importance prise par certains RSNP a attiré notre attention et nous a incité à mener une observation participante au sein du site web leader en la matière. Nous nous y sommes présenté sous notre véritable identité et avons commencé à réseauter, en intégrant progressivement les pratiques propres au milieu; le terrain d'étude se prêtant particulièrement à une démarche de type recherche-action. Après plus d'une année d'analyse minutieuse, nous avons cherché à mettre au jour des éléments d'interactions qui donnent à voir comment des individus cherchent à se dé-marquer (on parle de personal branding) dans un environnement concurrentiel et en ligne. Nous supposons que c'est un contexte pluriel (crise économique, réseaux sociaux spécialisés, mutations sociales...) qui a influencé l'adoption de certaines pratiques de gestion d'identité numérique à des fins professionnelles. Ce que nous cherchons à révéler plus particulièrement dans cet article, c'est que des individus inscrits sur de tels réseaux sociaux utilisent des techniques d'influence sociale, autrefois surtout appliquées dans le monde de l'entreprise, du marketing, et de la vente. Pour cela, nous allons nous appuyer sur quatre moments qui nous apparaissent comme particulièrement stratégiques dans ce qu'il convient d'appeler froidement le développement d'une politique de marketing relationnel de la personne.

\section{La création d'un profil attractif}

Entrer dans une démarche de développement de marque personnelle nécessite, en premier lieu, la création d'un compte utilisateur puis d'une page web spécifique, un « profil ». Plutôt que ce terme, celui de face pourrait être plus approprié. Rappelons en effet que Goffman définit « le terme de face comme étant la valeur sociale positive qu'une personne revendique effectivement [...] (Goffman 9). Pour le sociologue, en situation d'interaction, les individus cherchent à se valoriser et à ne présenter que les aspects les plus à même d'attirer l'admiration ou bien la sympathie. Il s'agit de jouer avec les apparences et de se présenter sous son meilleur jour. Transposée dans l'univers du marketing, la notion de face peut trouver quelque analogie avec le terme facing, qui exprime l'idée de préservation d'un ordre visuel et de mise en avant d'éléments, selon certaines priorités.

D'une certaine façon, il en va de même pour ce qui concerne l'affichage d'un profil LinkedIn qui donne à voir en premier lieu la photographie des inscrits, si ces derniers ont accepté de la fournir. Le texte, d'ordre argumentatif, vient ensuite. Certains témoignages tendent à montrer que cette question est sensible. Faut-il céder à l'idée de se montrer à des inconnus et si oui, de quelle façon?

La question de l'apparence dans un contexte de persuasion n'est pas récente.

Lévaluation du travail d'un individu en fonction de son attrait physique a été étudiée expérimentalement. Landy et Sigall avaient conçu un protocole dans lequel des étudiants devaient évaluer la qualité d'un travail écrit. La 
photographie de la jeune femme rédactrice du travail était jointe au texte dans deux des trois conditions expérimentales. Le premier groupe visualisait une personne perçue comme physiquement attrayante, le second visualisait une personne perçue comme n'étant pas physiquement attrayante. Les résultats de cette expérience ont montré que les étudiants fournissaient tendanciellement une évaluation plus positive du travail écrit lorsqu'il était présenté comme étant la production d'une personne physiquement attrayante. Cette étude est intéressante mais elle présente deux limites importantes dans le contexte qui nous intéresse : elle ne se situe pas dans un environnement professionnel, et de plus, les soixante étudiants sujets de l'expérience étaient tous de sexe masculin.

L'évaluation d'un texte argumentatif écrit en fonction de la qualité de la photographie de son auteur a aussi été étudiée, d'une façon similaire. Pallak présentait un texte à deux groupes d'étudiantes. Le premier groupe (condition de saillance haute) recevait, avec le texte, une photographie couleur, de bonne qualité, montrant un jeune homme jugé comme étant beau. Le second groupe (condition de saillance basse) recevait, avec le texte, une copie en noir et blanc, de mauvaise qualité, de la même photographie. Les résultats de cette recherche expérimentale ont montré des effets qui vont dans le sens de l'exemple précédent : les individus qui recevaient la photographie de bonne qualité acceptaient plus favorablement l'opinion défendue par le texte, sans réellement prendre en compte les arguments eux-mêmes, alors que les individus exposés à une photographie de piètre qualité traitaient les arguments sans considérer l'image.

Les éléments qui viennent d'être présentés tendent à montrer à quel point il est important, pour celui qui choisit sciemment de devenir une «personne marque ", de soigner la photographie qui va être choisie pour se présenter. Celle-ci, avant même de produire une influence sur la lecture qui sera faite de ce qui s'apparente à un $\mathrm{CV}$, aura un impact sur la visibilité de la page web de l'utilisateur. En effet, d'après Nicole Williams ${ }^{3}$, le fait d'ajouter une photographie à un profil multiplie par 7 son nombre de visites. D'après d'autres études qui semblent converger, il semble exister des types de photographies qui produisent un effet sur l'apparence de compétence, de sympathie, de leadership. Un regard dégagé (sauf à considérer le port de lunettes de vue qui donnerait, selon le stéréotype, une perception d' « intellectuel»), un sourire qui laisse percevoir les dents, le tout cadré en plan américain ou en plan poitrine, produiraient les effets les plus positifs.

Ces éléments sont là pour montrer à quel point le " packaging » demeure extrêmement important, qu'il s'agisse de mettre en avant une "marque personne » ou une «personne marque ».

3 Nicole WILLIAMS, «Your LinkedIn playbook: Tips for tackling the world's largest professional networking site ». LinkedIn Official Blog, 2012. Disponible sur http://blog.linkedin.com/2012/10/22/your-linkedinplaybook/, consulté le 28 janvier 2016. 


\section{La création de liens}

Dans une optique marchande, marketing, voire sociologique, il semble légitime de parler de développement de capital social pour qualifier un des intérêts qu'il y aurait à réseauter. Pour Bourdieu, le capital social permet de démultiplier le capital humain ou culturel. Degenne et Forsé développent cette notion en montrant que le capital social d'un individu n'est pas uniquement proportionnel au nombre de ses relations. Leur qualité est elle-même extrêmement importante et le fait d'avoir des relations qui ont du pouvoir semble plus important que leur nombre brut. En outre, si les liens forts (en matière de durée de relation, d'intimité, d'intensité émotionnelle, ou bien d'échange de services) sont précieux, les liens faibles (Granovetter) le sont eux aussi, notamment en ce qu'ils permettent d'accéder à d'autres réseaux que le sien. Enfin, on peut citer la notion d' "intermédiarité », que les auteurs jugent nécessaire pour analyser et bien appréhender certaines logiques de pouvoir. Par exemple, un individu central, y compris dans un cas de centralité faible (un intermédiaire faiblement connecté aux membres du réseau considéré), peut mettre à profit sa position d'intermédiaire en contrôlant la circulation de l'information, et tirer un pouvoir de sa position dans la structure.

Ces éléments pertinents pour le chercheur ou l'analyste ne sont pas nécessairement appréhendables aisément pour les usagers des réseaux sociaux numériques (Boutin et Amato). Pourtant, il semble que ces derniers aient progressivement acquis des pratiques qui leur permettent d'optimiser leurs interactions au sein de ces dispositifs et qu'ils en acquièrent implicitement, pour certains, des stratégies de réseautage relativement efficaces.

Dans un premier temps, la plupart des individus, peu aguerris aux codes et usages de cet espace numérique, préferent se constituer un réseau constitué d'autres individus avec lesquels existaient préalablement des liens forts. Ce seront par exemple des amis, des collègues de travail, des anciens camarades d'école, etc. Cette base de départ est intéressante car elle permet de ne pas se voir refuser une invitation, ce qui serait déjà décourageant. Mais il faut aussi savoir que le système limite, jusqu'au blocage, les possibilités d'invitation des individus trop souvent éconduits via un simple clic de souris sur le lien «Je ne connais pas cette personne ». Cette possibilité est à considérer avec précaution pour celui qui veut réseauter de façon intensive ; il lui faut faire preuve de prudence pour éviter cette éventualité qui pourrait lui être préjudiciable.

Passée cette étape, il devient difficile de savoir qui inviter. Le système propose un service qui permet, après autorisation de l'usager, d'importer le carnet d'adresses courriel présent sur son ordinateur afin d'inviter automatiquement les personnes qui y sont présentes. Cette pratique, parfois proposée au cours de la navigation de l'apprenti réseauteur, peut être acceptée machinalement et c'est ainsi qu'il en vient à spammer, plus ou moins à son insu, ceux qui constituent sa base de contacts. 
Plus ou moins conscients que le nombre de contacts d'un réseau est tout de même important, certains affichent, juste après leur identité, la mention «LION». Cela signifie «LinkedIn Open Networker». Il s'agit ici d'inciter les invitations d'individus parfaitement inconnus en leur indiquant ainsi qu'ils ne seront pas rejetés. Les LIONs affichent un nombre de contacts impressionnant mais il est permis de douter de la valeur de leur réseau, une observation fine de ces comptes montrant en effet une très grande hétérogénéité de la qualité de leurs membres. Toujours est-il qu'un nombre de contacts élevé semble synonyme de pouvoir. Mais ce nombre n'est pas toujours visible. Un individu qui compte plus de 500 contacts affiche sur son profil la mention « + de 500 relations». Il semble néanmoins que cette mention soit souvent un objectif à atteindre pour le réseauteur qui affiche ainsi son passage dans une autre dimension.

Pour notre part, nous avons opté pour une stratégie différente. L'hypothèse forte qui a sous-tendu notre action est que, dans un contexte de web de flux, ou bien d'attention distribuée ou non soutenue, l'individu a recours à un traitement heuristique (vs. systématique) de l'information, ce qui entraîne certains biais cognitifs (lire Amato et Boutin). Nous avons pensé dans ce cadre, après un filtre selon certains critères basiques (CSP, lieu d'habitation, etc.), inviter des individus de $2^{\mathrm{e}}$ niveau et négliger ceux de $3^{\mathrm{e}}$ niveau, afin de raccourcir au maximum le chemin possible permettant de se relier, au travers des réseaux relationnels respectifs. En outre, un des points majeurs de notre stratégie a été d'inviter préférentiellement des personnes avec lesquelles nous avions soit un maximum de relations en commun, soit une relation «importante ", même si inconnue(s) en réalité. L'idée était d'activer, chez la personne percevant ce fait, une heuristique biaisée du type « Si nous avons tant de relations communes, c'est que nous devons faire partie d'un même groupe social. Il se peut même que j'ai déjà réellement rencontré cette personne et que je ne m’en souvienne pas, toujours est-il que cela me donne envie d'accepter son invitation » et ainsi d'induire un sentiment de proximité. En procédant ainsi, nous avons pu observer une forme de corrélation entre nombre de contacts en commun et taux d'acceptation aux invitations. Certains messages reçus sitôt l'invitation acceptée nous donnent à penser que l'élément saillant utilisé ici était bien remarqué en tant que tel. Voici deux exemples de messages que nous avons pu ainsi recevoir, qui illustrent bien le raisonnement précédent :

- Merci ! Nous avons en commun 18 relations! Et votre nom m'est connu mais je ne sais si nous nous sommes croisés, bien à vous, Pauline Polly ${ }^{4}$.

- Bonsoir Stéphane, Merci pour votre demande de contact LinkedIn et soyez le bienvenu dans mon réseau. J'ai vu que nous avions, entre autres, Jean-Paul Canurri en commun, personne pour qui j'ai une grande estime. Au plaisir d'échanger sur nos activités respectives. Bien cordialement, Nicole.

4 Afin de préserver l'anonymat des personnes concernées, leur identité est modifiée tout au long de cet article. 


\section{L'attribution de compétences}

Précédemment, nous avons pu parler de " relations qui ont du pouvoir » ou bien de « relation importante " sans nécessairement expliciter ce que nous entendions par là. L'idée de pouvoir est souvent associée à celle de capacité d'action. La lecture d'un profil LinkedIn permet d'inférer des capacités d'une personne en fonction des postes qu'elle a occupés, des diplômes qu'elle a obtenus... Dans ces cas, le pouvoir est transféré par une instance de légitimation reconnue (une multinationale, une grande école prestigieuse...). Mais sur ce RSNP, il existe une autre façon de visibiliser efficacement son pouvoir présumé. Il s'agit de s'auto-attribuer jusqu'à 50 compétences relatives à ses propres domaines d'expertise. Par la suite, les relations de 1er niveau peuvent recommander tout ou partie de ces compétences. S'il est facile de demander à des personnes avec lesquelles on entretient des liens forts de recommander certaines compétences, cela l'est moins avec les autres. Pourtant, il semble socialement désirable de donner à voir la rubrique Compétences abondamment garnie de très nombreuses recommandations, en provenance d'individus extrêmement variés. Cet état de fait semble être particulièrement intégré par les usagers du réseau et ils en viennent, là encore, à mobiliser des stratégies efficaces dans cette optique. Dans une perspective de jugement heuristique (cf. supra), ces dernières peuvent être considérées comme un levier d'influence très puissant, agissant sur les attitudes et les représentations des visiteurs de la page web richement recommandée. En effet, pour Cialdini, dans de nombreuses situations, le jugement manifesté par de nombreux individus affecte notre propre jugement vis-à-vis du même objet, par une sorte d'effet de mimétisme. Il entraîne une forme d'adhésion automatique, sans que la réflexion ne soit convoquée.

Pour entraîner des recommandations de personnes avec lesquelles des liens faibles sont créés, les réseauteurs accoutumés se livrent à une forme d'économie du don et du contre-don (Mauss). Certains d'entre eux visitent le profil d'un inconnu et recommandent quelques compétences, apparemment dans l'attente d'un retour du même ordre, sans qu'aucune communication autour de cet acte n'ait lieu. Interpellé par cette pratique, nous avons visité les profils de personnes inconnues de nous, puis recommandé aléatoirement certaines de leurs compétences. La plupart d'entre elles nous a ensuite visité pour nous rendre la pareille, favorisant ainsi par là-même notre visibilité en renforçant aussi notre crédibilité perçue. Si cette pratique se fait souvent de façon très discrète, certains sont beaucoup plus explicites dans leur approche. Voici un des messages que nous avons reçus. Celui-ci illustre sans doute au mieux comment peut se réaliser une pratique plus directe. Juste auparavant, l'expéditeur venait de nous gratifier de 10 recommandations :

Dear colleague Stephane, Thank's for accept me. I really appreciate that. In the last time $i$ focused my work on some social themes. Would be my pleasure if you find 15-20 minutes to read my general informations on profile and my 4 posts. Your opinion will be more than 
wellcome, as much as your comment. I need opinions by experts; some of them you can already find in comments on my posts. Also, I will bighly appreciate if you find a minute of your time to endorse my 4 skills regarding mentioned fields of research: "Social psychology, "Ethics, "Diplomacy, "Child abuse prevention. Thank's \& best regards, Dr. Melio Horvath.

Cet exemple est réellement intéressant car il met au jour la mise en ouvre d'une autre et bien connue technique d'influence : la porte-au-nez (Cialdini, Vincent, Lewis, Catalan, Wheeler et Darby). Si l'on observe la requête, on s'aperçoit qu'elle se décompose ici en 3 temps :

- Le premier consiste à inviter un individu qui peut accepter par un simple clic de souris. Cet acte anodin peut être considéré comme un acte préparatoire, chez l'individu qui accepte l'invitation, si l'on considère les travaux sur la communication engageante et la communication numérique engageante (lire notamment Bernard et Joule 2004, Bernard et Joule 2005, Bernard, Halimi-Falkowicz et Courbet, Amato, Courbet, Bernard, Joule, Halimi-Falkowicz et Guéguen). Par ce simple clic, qui conditionne pourtant la suite de l'interaction, il y a ouverture d'un cours d'action favorisant la production d'actes plus coûteux et allant dans le même sens, ultérieurement. Mais ce n'est pas à ce stade que l'on peut parler de porte-au-nez;

- Le second temps invite à une lecture relativement rébarbative de 4 posts de l'auteur. La requête nous était apparue comme trop coûteuse pour pouvoir être acceptée ;

- Le troisième temps suit immédiatement au sein du même message. Il s'agit d'une requête de moindre coût puisqu'il suffit en définitive de produire 4 clics, très rapidement.

L'articulation du second et du troisième temps peut permettre de qualifier une tentative de communication d'influence qui peut s'apparenter à une forme de porte-au-nez, même si de façon non canonique, et c'est ainsi qu'à contre-cœur, nous avons apporté notre caution à un inconnu, autour de domaines pour le moins sensibles. Pour expliquer un des aspects de cette interaction, on peut faire appel à la norme de réciprocité qui, trivialement, peut se résumer ainsi : "vous accordez des avantages à ceux qui vous en ont consenti (Gouldner).

Nous avons pu observer de nombreuses pratiques de ce type sans que l'on puisse imaginer que tous les réseauteurs rencontrés avaient des connaissances théoriques précises permettant ce jeu d'influence et de co-influence. En revanche, il est permis d'imaginer que l'espace numérique considéré crée les conditions d'une forme de «darwinisme social » permettant aux plus aptes de saisir instinctivement les règles du milieu et de maximiser leurs pratiques en fonction.

5 Cette technique consiste à formuler une première requête trop coûteuse pour pouvoir être acceptée, avant d'en formuler une seconde, bien moins coûteuse, portant sur le comportement réellement attendu. 


\section{L'animation communautaire et le renforcement des liens}

Le profil est désormais créé, une base de liens forts a servi pour les premiers développements, les liens faibles (invitations lancées, invitations reçues et acceptées) se sont multipliés. Pour notre part et après un peu plus d'une année, cela nous a conduit à dépasser le nombre de 30000 abonnés, ce qui paraît considérable. Notre profil fait partie du « top $1 \%$ des vues de profil» (classement international), ce qui a pu nous conduire à dépasser certaines personnalités du monde politique ou de l'entreprise, pourtant internationalement connues et fortement médiatisées. Comble du raffinement, le RSNP a intégré dans son système un outil qui permet d'évaluer sa capacité à bien se vendre et à situer sa pratique parmi celle des autres réseauteurs. Voici ce que dit LinkedIn à propos de cet index : « Le Social Selling Index (SSI) mesure votre efficacité à imposer votre marque professionnelle, à trouver les bonnes personnes, à communiquer avec les bonnes infos et à construire des relations. » Les 4 dimensions citées sont chacune scorées et permettent d'obtenir un indice global plafonné à 100. Pour notre part, un SSI de 77/100 nous place dans le top $1 \%$ des individus de notre secteur (Enseignement supérieur) dans lequel le SSI moyen est de 17/100. Voilà qui peut motiver certains mais voilà aussi de quoi en inquiéter bien d'autres. Désormais, le pouvoir, les qualités d'un individu peuvent se trouver évalués par un algorithme. Il semble que les organisations n'échappent pas à ce type d'évaluation puisque désormais, LinkedIn se propose aussi de classer les universités6, et certaines d'entre elles mobilisent leurs étudiants pour être présents sur ce réseau. Il n'est pas utopique d'envisager de prochains cours de personal branding au sein de ces mêmes établissements. Des formations dédiées existent déjà pour les professionnels qui deviennent alors des ambassadeurs de leur entreprise, à peu de frais. Voilà de quoi s'interroger sur les implications sociales ou organisationnelles de ces nouvelles pratiques, dans la lignée de certaines réflexions telles que celles de Evgeny Morozov.

Toujours est-il que le dispositif incite à " développer son réseau " (via un lien spécifique) et encourage à optimiser ses pratiques relationnelles (cf. SSI). On en revient à l'idée de développer des liens faibles et à renforcer les liens déjà existants. Pour ce faire, il est possible de devenir une sorte d'animateur de communauté (community manager) en intervenant sur le flux d'information produit par ses abonnés. Il est possible d' « aimer » une information par un clic, ce qui constitue une action de visibilité minimale, mais aussi d'y apporter un commentaire, ou de la partager avec toutes ses relations. Il est aussi possible de diffuser une information présente sur un autre site web ou bien encore de devenir producteur d'information originale en «publiant un post ». Ces actions répétées, si bien calibrées en fonction de ses relations, réalisées « au bon moment» (il ne s'agit pas de relayer une information datée mais

6 Benoît FLOC'H, 2016, «LinkedIn veut secouer les classements des universités». Le Monde Campus. Disponible sur http://www.lemonde.fr/campus/article/2016/01/06/linkedin-le-classement-d-universites-quiva-tout-changer_4842227_4401467.html, consulté le 31 janvier 2016. 
plutôt d'être le premier à la transmettre) permettent d'entretenir un contact, de se rendre familier, de renforcer les liens existants, voire de diffuser un sentiment d'expertise autour de thématiques. De façon plus prosaïque, ce type d'action permet d'assurer une promotion efficace. À titre d'exemple, lorsque nous «partageons une nouvelle » avec nos relations, celle-ci a été vue en moyenne 6000 fois au bout de 3 à 4 jours, mais aussi «aimée», « commentée», «partagée». C'est dire si une utilisation fine du dispositif peut s'avérer puissante, en matière de notoriété et d'image, et ce toujours à moindre coût. Afin d'illustrer le sentiment que peut donner un tel profil, voici un message que nous avons reçu ${ }^{7}$ de la part d'un consultant en marketing réputé dans son domaine:

Bonjour Stéphane, $[\ldots]$ votre profil d'ex consultant en com' revenu aux SIC m'a interpellé $[\ldots]$ et vous m'avez intéressé encore plus quand j'ai vu votre nombre de contacts à 27.000+. Je suis évidemment convaincu que tous vos contacts apprécient votre expertise, mais ce compteur stratosphérique m'intrigue d'autant plus que je veux développer mon réseau, quitte à utiliser des scripts / macros mais toujours de façon "clean" pour automatiser certaines actions. D'où deux questions : - quelle stratégie à la fois "quali" \& quanti recommandez-vous ? Si vous avez deux minutes pour échanger sur ça, je prends. [...] Votre éclairage / expérience m’intéresse. J’ai déjà vu d'autres profils à $80 \mathrm{~K}$ ou $100 \mathrm{~K}$ contacts, mais le vôtre est atypique. Au plaisir de vous lire, en vous souhaitant bon succès et bonne année 2016. [...] Jean Pasquier / mob 06 XX XX XX XX / jean@pasquiermarketing.com

\section{Conclusion: Des réseaux d'influence à l'influence sur les réseaux}

Dans un temps pas si éloigné, pour se rapprocher d'une personne de pouvoir, pour trouver un emploi, pour développer un nouveau marché, une des possibilités était de se voir introduit ou appuyé (on disait " pistonné») par un ou plusieurs membres de groupes influents. La société en regorge (lobbies, syndicats...). L'avènement de la Société en réseaux (Castells) a largement contribué, entre autres, à reconfigurer certaines pratiques sociales, ou bien à favoriser l'individualisation des tâches et des trajectoires professionnelles. La « digitalisation de la société » effraie ceux qui craignent une fragmentation du monde du travail et remplit d'enthousiasme ceux qui savent rapidement tirer parti des dernières innovations et qui savent lire les mouvements en cours de création, pour en tirer quelque opportunité. C'est cette époque qui voit l'essor des RSNP dans lesquels l'individu a le sentiment, en toute liberté et sans même en être choqué, de devenir une marque comme les autres. Il n'est alors pas anormal qu'il en vienne à se comporter en tant que telle, en adoptant certains de ses codes et de ses pratiques. L'individu en vient même à adopter des stratégies de communication d'influence d'une grande efficacité, et cela

7 Nous avons aussi reçu des propositions de collaboration (animation de formations, coaching en personal branding, enable branding, growth hacking, inbound marketing, social selling, brand advocacy... ). 
presque « naturellement » puisqu'il ne les a pas étudiées. Nous postulons que des tensions inhérentes à cette période de transition ont su réveiller certaines aptitudes primordiales où la vie sociale prend toujours forme autour d'enjeux d'influence et de co-influence.

Dans cet article, le propos nétait pas de révéler l'ensemble des stratégies mises au jour chez les acteurs pendant notre observation participante (il y aurait bien plus à dire!) mais d'amener à une réflexion pour que nous comprenions au plus vite certaines mutations alors quelles sont encore en train de se produire. Il ne s'agit donc pas de faire du marketing de soi mais plutôt de révéler certaines pratiques d'influence, en situation de communication, qui ont cours sur les RSNP pour que nous puissions nous en prémunir, regagner en autonomie et agir en acteurs responsables. Il nous est apparu qu'il s'agissait là d'une problématique d'importance à ouvrir en Sciences de l'Information et de la Communication, dans le cadre d'une « interdisciplinarité sans tabou ni censure-autocensure » (Bernard 53).

\section{BIBLIOGRAPHIE}

AMATO Stéphane (2013), Communication numérique engageante : relations entre théories, méthodologies et terrains, Aix-Marseille Université, Thèse de doctorat en Sciences de l'Information et de la Communication.

AMATO Stéphane et BOUTIN Éric, 2013, «Étude des effets d'ordre dans la recherche d'information sur le Web : le cas d'une expérimentation sur les techniques de sevrage tabagique», In ESSACHESS - Journal for Communication Studies, 6 : 1, p. 57-73.

BERNARD Françoise, 2015, «Les théories de l'influence en communication», in Hermès, 71, p. 45-57.

BERNARD Françoise, HALIMI-FALKOWICZ Séverine et COURBET Didier (octobre 2010), «Expérimentation et communication environnementale : la communication engageante et instituante», dans D. Courbet, Objectiver l'bumain? Volume 2. Communication et expérimentation, Paris, Lavoisier, 71-113.

BERNARD Françoise et JOULE Robert-Vincent, 2004, « Lien, sens et action : vers une communication engageante », in Communication \& Organisation, 24, p. 347-362.

BERNARD Françoise et JOULE Robert-Vincent, 2005, «Le pluralisme méthodologique en sciences de l'information et de la communication à l'épreuve de la "communication engageante"», in Questions de communication, 7, p. 185-208.

BOURDIEU Pierre (1980), «Le capital social: notes provisoires», dans Actes de la Recherche en Sciences Sociales, 31, 2-3.

BOUTIN Éric et AMATO Stéphane (2013), «Le web interactionniste: représentations et réalité", dans Actes du colloque: Contextes, langues et cultures dans l'organisation des Connaissances, Paris, ISKO France, 267-280.

CASTELLS Manuel (2001), La société en réseaux. Lère de l'information, Paris, Fayard, $671 \mathrm{p}$. 
CIALDINI Robert B. (2001), Influence: Science and Practice, Boston, Allyn and Bacon, $262 \mathrm{p}$.

CIALDINI Robert B., VINCENT Joyce E., LEWIS Stephen K, CATALAN José, WHEELER Diane et DARBY Betty Lee, 1975, «Reciprocal concessions procedure for inducing compliance: The door-in-the-face technique», in Journal of Personality and Social Psychology, 31 : 2, p. 206-215.

COURBET Didier, BERNARD Françoise, JOULE Robert-Vincent, HALIMIFALKOWICZ Séverine et GUÉGUEN Nicolas, 2016, « Small clicks, great effects: the immediate and delayed influence of websites containing serious games on behavior and attitude ", in International Journal of Advertising, à paraître.

DEGENNE Alain et FORSÉ Michel (2004), Les réseaux sociaux, Paris, Armand Colin, $296 \mathrm{p}$.

GOFFMAN Erving (1974), Les rites d'interaction, Paris, Les éditions de minuit, $231 \mathrm{p}$. gouldner Alvin W., 1960, « The norm of reciprocity: A preliminary statement ", in American Sociological Review, 25 : 2, p. 161-178.

GRANOVETTER Mark S., mai 1973, "The strength of weak ties ", in American Journal of Sociology, 78 : 6, p. 1360-1380.

LANDY David et SIGALL Harold, mars 1974, « Beauty is talent: Task evaluation as a function of the performer's physical attractiveness ", in Journal of Personality and Social Psychology, 29 : 3, p. 299-304.

MAUSS Marcel (2012), Essai sur le don, Paris, Presses Universitaires de France, 252 p.

MOROZOV Evgeny (2014), Pour tout résoudre cliquez ici - l'aberration du solutionnisme technologique, Limoges, Fyp éditions, 352 p.

PALLAK Suzanne, 1982, « Salience of a communicator's physical attractiveness and persuasion: A heuristic versus systematic processing interpretation », in Social Cognition, $2: 2$, p. $156-168$

Résumé: Cet article est le fruit d'une observation participante de plus d'un an sur le réseau social numérique professionnel LinkedIn. L'auteur s'est placé dans une situation de développement de marque personnelle en ligne. Au fil du temps, il a identifié 4 temps forts de cette démarche qui sont décrits sommairement : la création d'un profil attractif, la création de liens, l'attribution de compétences, l'animation communautaire et le renforcement des liens. Une observation fine des pratiques de réseautage dans cet espace numérique permet de révéler de forts enjeux d'influence et de co-influence. Ces derniers sont analysés et questionnés. Enfin, l'auteur de cette recherche modère l'ambition de son travail qui se veut avant tout exploratoire et propose de pousser la réflexion ultérieurement. Mots-clés : réseaux sociaux numériques professionnels, LinkedIn, Marque personnelle, Réseautage, Communication d'influence, Influence sociale.

Abstract: This article is the product of a participant observation on the professional digital social network LinkedIn over more than a year. The author put himself in a situation of online personal branding. Over time, he identified 4 highlights of this process, which are described 
summarily: the creation of an attractive profile, the creation of links, skills and expertise endorsements, community management and strengthening ties. A keen observation of these networking practices in this digital space reveals strong influence and co-influence stakes. The latter are analyzed and challenged. At last, the author of this research mitigates the ambition of his work which he intends as exploratory and suggests to continue the study later on.

Keywords: professional digital social networks, LinkedIn, Personal branding, Networking, Communication influence, Social influence. 